\title{
Four-wave mixing excitations in a dissipative polariton quantum fluid
}

\author{
V. Kohnle, ${ }^{1}$ Y. Léger, ${ }^{1,2, *}$ M. Wouters, ${ }^{3}$ M. Richard, ${ }^{4}$ M. T. Portella-Oberli, ${ }^{1}$ and B. Deveaud ${ }^{1}$ \\ ${ }^{1}$ Institute of Condensed Matter Physics, École Polytechnique Fédérale de Lausanne, CH-1015 Lausanne, Switzerland \\ ${ }^{2}$ FOTON Laboratory, Université Européenne de Bretagne, CNRS-INSA-UR1, F-35708 Rennes, France \\ ${ }^{3} T Q C$, University of Antwerp, Universiteislpein 1, 2610 Antwerpen, Belgium \\ ${ }^{4}$ Nano-Physics and Semiconductor group, Néel Institute, CNRS Grenoble, France
}

(Received 27 April 2012; revised manuscript received 19 June 2012; published 8 August 2012)

\begin{abstract}
The Bogoliubov transformation of a polariton quantum fluid has recently been revealed in the four-wave mixing response of a driven microcavity polariton gas. In this work, we investigate the modifications that the dual nature of microcavity polaritons produce on the excitations of this particular half-light-half-matter quantum fluid. We discuss in particular the Bogoliubov character of the excitations of a lower polariton superfluid when it coexists with upper polaritons. We show unique effects resulting from the interplay between polariton decay and Bogoliubov transformation such as the modification of the Bogoliubov dispersion or the slowing down of the four-wave mixing dynamics.
\end{abstract}

DOI: 10.1103/PhysRevB.86.064508

PACS number(s): 67.10.-j, 71.36.+c, 78.47.nj, 78.67.De

\section{INTRODUCTION}

When the particles of a bosonic quantum fluid are submitted to repulsive interactions, the transition to quantum degeneracy is accompanied by a transformation of the fluid elementary excitations. ${ }^{1}$ This so-called Bogoliubov transformation is at the basis of the superfluid behavior. During the last 15 years, most of the Bose-Einstein condensates that could be realized, both with cold atoms and in the solid state, have shown clear signs of superfluidity such as frictionless flow $^{2-4}$ or quantized vortices. ${ }^{5-8}$ Still, the most convincing demonstration of superfluidity remains the measurement of the excitation spectrum of the Bose gas. This spectrum should not only show a phononlike behavior ${ }^{9,10}$ but also the appearance of a negative energy dispersion, often called "ghost branch," resulting from the prevalence of parametric processes in the excitation of the quantum fluid. ${ }^{11}$ Such a negative energy resonance has been first observed by Vogels et al. with condensates of sodium atoms in a magnetic trap. ${ }^{12}$ Recently, the Bogoliubov dispersion has been investigated in the solid state in a coherent polariton gas, ${ }^{13}$ and the ghost dispersion branch has finally been observed. ${ }^{14}$

Microcavity polariton fluid is extremely different from other bosonic quantum fluids as polaritons originate from the strong coupling of quantum-well excitons with cavity modes of semiconductor microcavities. The main consequence of this unique nature is the short lifetime of the particles in the 10 -ps range. This nonequilibrium nature is at the same time a great advantage and a drawback for polariton condensation: On the one hand, dissipation has sometimes been raised as an obstacle for Bose-Einstein condensation, which requires thermal equilibrium; on the other hand, dissipation offers a unique way to probe the quantum fluid through the photons emitted out of the cavity. More than this, some of the most interesting dynamics observed in polariton condensates result from nonequilibrium. ${ }^{15-17}$ Stabilization of polariton superflows by dissipation has even been predicted. ${ }^{18}$

Strong-coupling regime brings another original aspect to the physics of microcavities: two polariton modes, sharing the same photonic and excitonic components can coexist in the cavity. So far, most of the efforts of the community have been concentrated on the physics of lower-energy polaritons (LP) as they constitute the ground state of the system, where spontaneous condensation is observed. ${ }^{19}$ However, the investigation of upper polaritons (UPs) is also valuable as the UPs are more affected than LPs by interactions with the excitonic states lying in the same energy range, causing inhomogeneous broadening in the UP mode. ${ }^{20}$

In this paper, we investigate the excitations of a coherent polariton gas by means of the four-wave mixing (FWM) technique. The Bogoliubov transformation was already discussed deeply in a previous paper. ${ }^{14} \mathrm{We}$ focus here on the temporal and wave-vector dependence of the FWM spectrum. We discuss in detail the strong effect of dissipation on the superfluid excitation spectrum resulting in the formation of replicas of the dispersion branches. We also investigate the interaction between lower and upper polaritons and its influence on the superfluid character of the lower polaritons. This work is organized as follows: In Sec. I, we introduce the physics of Bogoliubov excitations from the general case to the specific case of polariton system. In Sec. II, we explain the FWM technique and the setup used for these experiments, while Sec. III is dedicated to the sample description. The main section IV reports on the experimental results obtained on Bogoliubov excitations. In a first part, we focus on the delay-time dependence of the FWM spectrum of the polariton quantum fluid. We study both the effect of dissipation and the interactions between LPs and UPs on the superfluid excitations. In a second part of Sec. IV, we discuss the effect of dissipation on the FWM momentum dispersion. Aside from the experimental results, we perform semianalytical simulations based on the Gross-Pitaevskii equation for comparison with our experimental findings.

\section{BOGOLIUBOV EXCITATIONS}

Repulsive interactions between bosons in a weakly interacting Bose gas change the low-momentum excitations of a quantum fluid from single particles to collective excitations showing a phononlike behavior. ${ }^{1}$ In this framework, superfluid excitations of a two-dimensional (2D) bosonic gas are 
(a) Bogoliubov picture

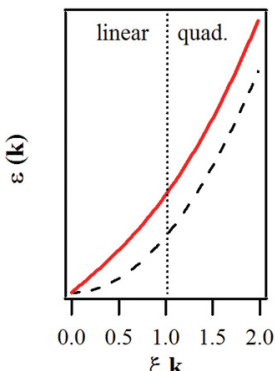

(b) Single-particle picture

(c) Experiment

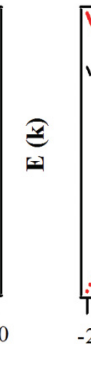

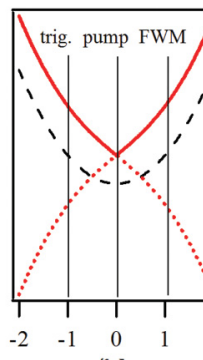

$\xi \mathbf{k}$

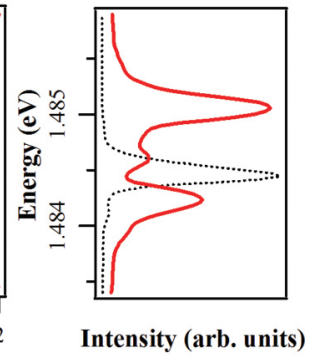

Intensity (arb. units)

FIG. 1. (Color online) (a) Momentum dispersions $\epsilon_{B}(\mathbf{k})$ of Bogoliubov quasiparticle excitations (red plain curve) and free-particle excitations (black dashed line) of a 2D boson gas $\left(\frac{\hbar^{2} k^{2}}{2 m}\right)$. The transition from the phononlike (linear) to the free-particle (quadratic) regime takes place at $k \approx \xi^{-1}$. The excitation spectra are renormalized to the superfluid energy. (b) In the single-particle picture, Bogoliubov excitations show up as a couple of symmetric dispersions around the blue-shifted superfluid energy as a result of the Bogoliubov transformation. (c) Experimental FWM response of a quiescent $(k=$ 0 ) polariton quantum fluid after perturbation at $k=1 \mu \mathrm{m}^{-1}$ (red line) revealing the normal and ghost branches of the Bogoliubov spectrum lying at 1485.1 and $1484.2 \mathrm{meV}$, respectively. The low-density $k=0$ resonance obtained from transmission measurements is plotted for comparison (black dotted line).

described as quasiparticles sometimes called "bogolons," the creation and annihilation operators $\alpha^{\dagger}$ and $\alpha$ of which read as

$$
\begin{aligned}
& \hat{\alpha}_{\mathbf{k}}=u_{\mathbf{k}} \hat{a}_{\mathbf{k}}+v_{\mathbf{k}} \hat{a}_{-\mathbf{k}}^{\dagger}, \\
& \hat{\alpha}_{-\mathbf{k}}^{\dagger}=u_{\mathbf{k}} \hat{a}_{-\mathbf{k}}^{\dagger}+v_{\mathbf{k}} \hat{a}_{\mathbf{k}} .
\end{aligned}
$$

These new operators are expressed in terms of the creation and annihilation operators $a^{\dagger}$ and $a$ for free particles in momentum basis. The bogolon is thus a coherent superposition of counterpropagating particle and hole. The energy $\epsilon_{B}(\mathbf{k})$ of such an excitation is given by

$$
\epsilon_{B}(\mathbf{k})=\sqrt{\left(\frac{\hbar^{2} k^{2}}{2 m}\right)\left(\frac{\hbar^{2} k^{2}}{2 m}+2 g n\right)},
$$

where $m$ is the single-particle mass, $g$ the repulsive interaction constant, and $n$ the superfluid density.

In Fig. 1(a), the excitation spectrum $\epsilon_{B}(\mathbf{k})$ of bogolons is displayed together with the free-particle quadratic dispersion. For small momenta $\hbar k<\xi^{-1}$, where $\xi=\sqrt{\frac{\hbar}{2 m g n}}$ is the healing length of the system, the quasiparticle dispersion becomes linear. This phononlike behavior, allowing us to define a critical velocity, is the cornerstone of superfluidity. However, in the single-particle dispersion spectrum [see Fig. 1(b)], the Bogoliubov transformation not only causes the linearization of the dispersion, but also the appearance of a negative energy ghost branch (GB) as a symmetrical image of the normal phononlike dispersion branch (NB) with respect to the condensate. ${ }^{21,22}$

Microcavity polaritons, for which superfluidity has first been demonstrated through frictionless measurements, ${ }^{3,4}$ appear as a model system to investigate the Bogoliubov excitation spectrum. ${ }^{13,14,23,24}$ These composite bosons show indeed unique properties inherited from both their components: a very

small mass from the photonic part and Coulomb mediated repulsive interactions from the excitonic one. A first attempt to measure the Bogoliubov dispersion in a spontaneous polariton condensate has been carried out through photoluminescence experiments. ${ }^{13}$ Our work is based on a different approach suggested by Wouters and Carusotto: the superfluid excitation spectrum can be probed in the single-particle basis through four-wave mixing processes. ${ }^{24}$ When a quiescent polariton condensate $(k=0)$ is submitted to an optical perturbation at a wave vector $k<\xi^{-1}$, Bogoliubov excitations are generated, resulting in light emission at the opposite wave vector $-k$. The FWM signal should reveal both normal and ghost branches of the Bogoliubov spectrum. Equivalently, this process can be described as a parametric scattering scheme. A pair of condensate polaritons is coherently scattered into a polariton at $\left(+E,+\mathbf{k}_{t}\right)$ and another one with symmetric wave vector and energy $\left(-E,-\mathbf{k}_{t}\right)$. The same applies at opposite energies: $\left[(0,0)+(0,0) \longrightarrow\left(-E,+\mathbf{k}_{t}\right)+\left(+E,-\mathbf{k}_{t}\right)\right]$.

However, the small condensate fraction and the influence of the excitonic reservoir ${ }^{25}$ can prevent the full formation of bogolons. Furthermore, for nonresonantly excited microcavities, the theory predicts as well a change in the equilibrium Bogoliubov spectrum: a flat excitation spectrum close to $k=0$ appears resulting from the gain-loss mechanism, making the observation of the Bogoliubov transformation at low $k$ difficult.

In order to probe the Bogoliubov transformation in the optimal conditions, we chose to develop a heterodyne version of the FWM scheme proposed by Wouters et al. In this case, the condensate is created resonantly so that its phase is fully controlled. Phase modulation of the pump pulse, creating the condensate, and of the second pulse, triggering the excitation, allows for a precise selection of the FWM signal through spectral interferometry. The first advantage of this method is that the FWM response can be separated from the condensate emission even at very small wave vector. The second advantage comes from the fact that resonant circularly polarized pulsed excitation limits the creation of a nonradiative exciton reservoir which could modify the dynamics of the quantum fluid. All the details of the heterodyne FWM experiment are provided in Sec. II.

With this experimental setup, we demonstrated in a previous paper the crossover between a dilute polariton fluid and a polariton superfluid with Bogoliubov excitations. ${ }^{14}$ In the superfluid regime, the FWM response shows two main emission peaks with comparable intensities and linewidths, which are the signatures of the normal and ghost branches of the Bogoliubov spectrum [Fig. 1(c)]. In the following, we concentrate on the time evolution of these excitations, revealing interactions between lower and upper polaritons. We also analyze the effects of dissipation on the polariton superfluid dispersion.

\section{HETERODYNE FOUR-WAVE MIXING EXPERIMENT}

Two-beam heterodyne FWM is based on spectral interferometry. This provides a unique way to select and amplify weak coherent contributions from all the light emitted by the system under scrutiny. ${ }^{26,27}$ This method is particularly suited to our study as bogolons are small contributions compared to the condensate emission, with close energies and momenta. 


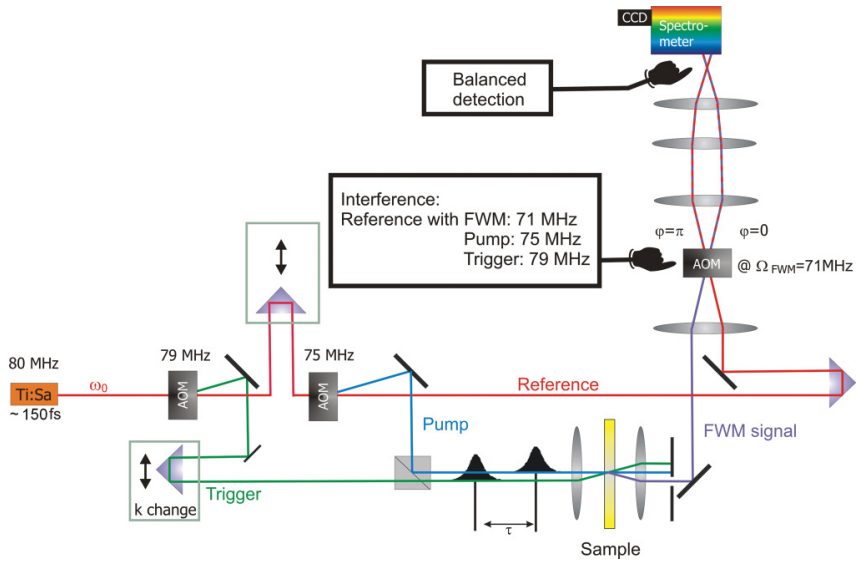

FIG. 2. (Color online) Two-beam heterodyne FWM: experiment.

The sketch of the heterodyne FWM setup that we developed is presented in Fig. 2. We use a pulsed Ti:sapphire laser emitting 125 -fs-long pulses (12-meV spectral width) at a rate of $80 \mathrm{MHz}$. The pulses are spectrally centered on the lower polariton $\mathbf{k}=0$ energy but cover the energy range of both lower and upper polariton dispersion curves. Our experiment requires three beams: a pump, a trigger, and a reference beam. To obtain the different beams, we use two acousto-optic modulators (AOMs) and use for each of them the zero- and first-order diffracted beams. The first-order deflected beam of the first AOM, which is driven at $\Omega_{t} /(2 \pi)=79 \mathrm{MHz}$, forms the trigger. It passes a retroreflector, the lateral translation of which corresponds to the change of the trigger beam incidence angle onto the sample. The nondeflected zero-order beam is used to form the pump and reference beams. It first passes through a delay line, which allows us to vary the pump-trigger delay time and then through a second AOM, driven at $\Omega_{p} /$ $(2 \pi)=75 \mathrm{MHz}$. The frequency-unchanged zero-order beam is taken as the reference, while the 75-MHz-shifted first-order diffraction forms the pump. The reference is not directed onto the sample, but used on the detection side to perform spectral interferometry by spatially and spectrally overlapping it with the FWM signal. The optical paths of trigger and pump are recombined through a cube and focused onto the sample. The pump hits the sample at normal incidence $\left(k_{P}=0\right)$. The trigger excites the sample with wave vectors between $k_{T}=0$ and $1.2 \mu \mathrm{m}^{-1}$. The pump intensity is high in order to ensure the formation of a dense polariton gas without saturating the polariton population (around $9 \times 10^{10}$ photons $/$ pulse $/ \mathrm{cm}^{2}$ ). The trigger pulse is 10 times weaker than the pump pulse. The sample is mounted in a helium 4 continuous flow cryostat and is kept at around $5 \mathrm{~K}$. The FWM signal is collected in transmission with a microscope objective having an numerical aperture NA $=0.5$. The FWM signal emitted in the direction $2 \mathbf{k}_{p}-\mathbf{k}_{t}$ is spatially filtered and directed into a mixing AOM together with the reference in such a way that the diffracted beam of the reference overlaps with the nondiffracted FWM signal and vice versa. The AOM, driven at $\Omega_{\mathrm{FWM}}=2 \Omega_{p}-\Omega_{t}=71 \mathrm{MHz}$, up-shifts the diffracted reference field frequency and down-shifts the diffracted signal field frequency by $\Omega_{\mathrm{FWM}}$. This results in the spectral overlap of reference and FWM fields and a $\pi$-phase shift for the two heterodyne channels. The mixed
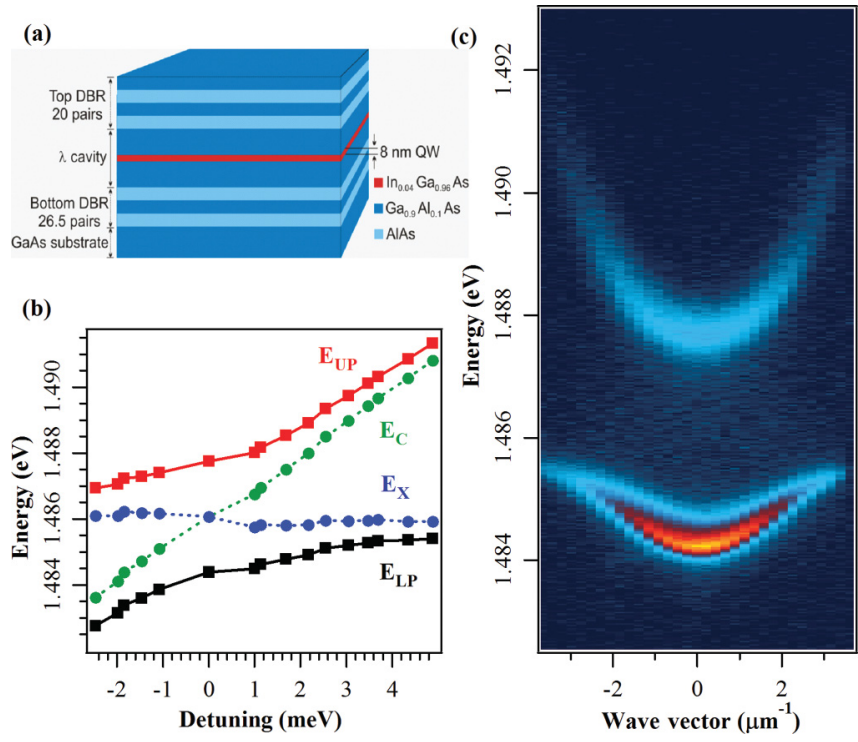

FIG. 3. (Color online) (a) Structure of the high-quality GaAsbased microcavity sample, consisting of two AlGaAs/AlAs Bragg reflectors with an embedded InGaAs quantum well. (b) Measured anticrossing curve as a function of the cavity detuning. (c) Polariton dispersion curve obtained in photoluminescence at zero detuning.

beams are then dispersed in a spectrometer and recorded with a nitrogen-cooled charge-coupled device (CCD). To recover the interference term between the FWM signal and the reference field only, we subtract the two $\pi$-shifted interferograms (balanced detection). In this way, the classical noise, e.g., photoluminescence or laser fluctuations, is largely suppressed. From the measured interference, we deduce the time-integrated signal in amplitude and phase by spectral interferometry. ${ }^{26}$ We define positive delay $\tau$ as the time ordering for which the pump pulse hits the sample before the trigger pulse. The reader will note that because we measure the FWM signal obtained from the combination $E_{\mathrm{FWM}} \propto E_{p}^{2} E_{t}^{*}$, the time ordering of the pulses at positive delays provides a 2 quantum signal in FWM terminology: a two-photon absorption is induced by the first pulse and during the delay between the two pulses, the system evolves at the frequency of this double excitation. At negative delays, one usually refers to a rephrasing signal (such as photon echoes in inhomogeneous systems) since the phase evolution of the FWM emission will be reversed compared to the one of the system after the first pulse, which evolves as $E_{t}^{*}{ }^{28}$

\section{SAMPLE}

The sample under investigation is a high-quality III-V GaAs-based microcavity. ${ }^{29}$ In Fig. 3(a), a scheme of the sample structure is presented. The sample was grown on a GaAs substrate. The GaAs $\lambda$ cavity, containing one 8-nm quantum well of $\operatorname{In}_{0.04} \mathrm{Ga}_{0.96} \mathrm{As}$, is sandwiched between a pair of distributed Bragg reflectors (DBRs), which are composed of alternated $\lambda / 4$ layers of $\mathrm{Ga}_{0.9} \mathrm{Al}_{0.1} \mathrm{As}$ and $\mathrm{AlAs}$. The bottom mirror contains 26.5 pairs and the top mirror consists of 20 pairs. The cavity spacer layer is wedged and thus the resonance frequency of the cavity can be varied by moving the laser spot over the sample [see Fig. 3(b)]. The properties of the quantum 


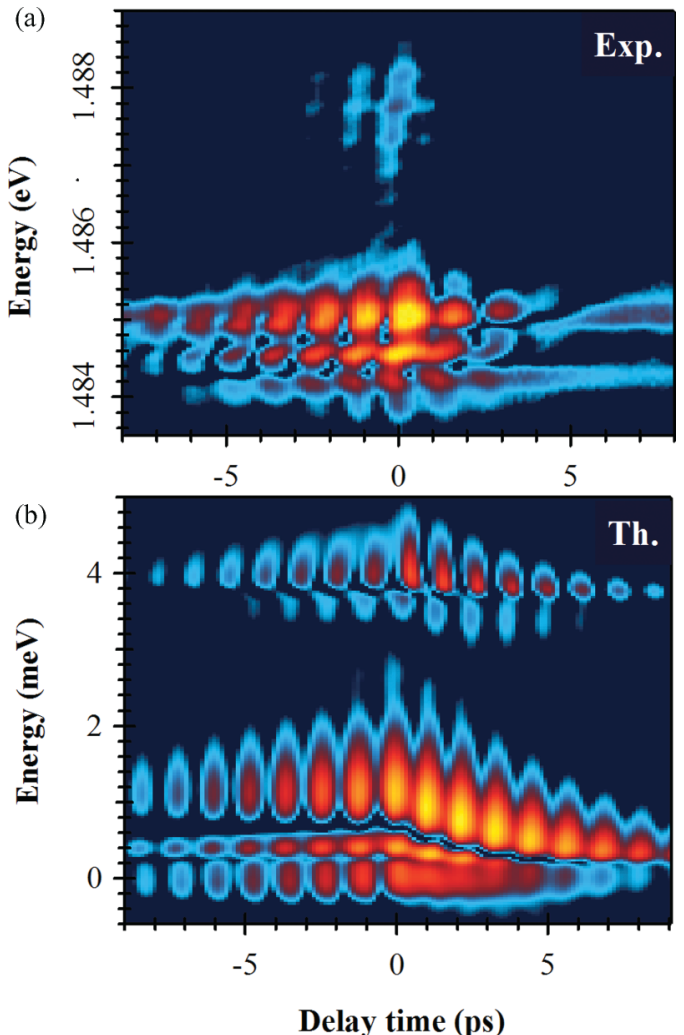

FIG. 4. (Color online) Experimental (a) and calculated (b) delay dependence of the FWM response for a perturbation of the polariton quantum fluid at $k=1 \mu \mathrm{m}^{-1}$ in log color scale.

well, which exhibit an exciton energy of $1.486 \mathrm{eV}$, are the same all over the sample. The Rabi splitting at zero detuning between the cavity and exciton mode is measured to be $\Omega_{R}=3.45 \mathrm{meV}$.

Figure 3(c) displays the measured polariton spectrum resolved along one direction of the momentum space $k_{\|}$ around zero detuning. This dispersion was obtained in photoluminescence under weak nonresonant excitation with a continuous-wave helium-neon laser $(632.8 \mathrm{~nm})$. The main parts of the FWM experiments are performed at this detuning. The LP and the UP branch dispersions are clearly observed.

\section{FOUR-WAVE MIXING SPECTRA}

The spectrum presented in Fig. 1(c) is the FWM response obtained at positive delay $\tau$ (integrated between 1.5 and 8 ps), when the condensate is created first and then perturbed by the trigger pulse, creating Bogoliubov excitations in the polariton quantum fluid. The spectrum shows clearly two main resonances, identified as the normal and ghost branches of the Bogoliubov spectrum, and a weak third emission peak between them. We now focus on the evolution of this spectrum when varying the delay between the pump and trigger pulse (Fig. 4). Both angle selection and heterodyning ensure that we detect only the third-order nonlinear emission from the polariton gas. The detected signal then depends on both the pump and the trigger pulses. The asymmetry of the pulse configuration (different incidence angles and excitation intensities) offers the opportunity to extract from the FWM delay dependence different behaviors depending on the time ordering of the pulses. The positive delay window provides information on the coherent evolution of the polariton quantum fluid, created by the pump pulse, whereas the FWM dynamics in the negative delay window corresponds to the nonlinear response of the low-density polaritons created by the trigger.

\section{A. Delay dependence}

If the appearance of the whole Bogoliubov spectrum with normal and ghost branches is quite intuitive for positive delays, the general delay dependence of the FWM response raises an interesting question: What should one expect when the small excitation at finite $k$ is created prior to the polariton quantum fluid? As a matter of fact, at negative delays, the trigger pulse interacts with the low-density normal dispersion. When the polariton quantum fluid is created at later time, the trigger polaritons should transform into a Bogoliubov excitation. One could naively expect FWM emission at a single energy position only, due to phase-matching selection rules, namely, on the ghost branch. The delay-dependent FWM spectrum presented in Fig. 4(a) shows on the contrary that normal and ghost branches coexist even on the negative delay side, at 1485.1 and $1484.2 \mathrm{meV}$, respectively.

The observed FWM emission of the normal branch at negative delays seemingly indicates a breaking of the energy conservation of the FWM process, but can simply be explained as follows: When the polariton quantum fluid is injected at $k=0$, polaritons from the trigger pulse, with wave vector $\mathrm{k}$, undergo the Bogoliubov transformation of the dispersion:

$$
a_{\mathbf{k}} \rightarrow u_{\mathbf{k}} \alpha_{\mathbf{k}}-v_{\mathbf{k}} \alpha_{-\mathbf{k}}^{\dagger} .
$$

A coherent superposition of two counterpropagating Bogoliubov excitations (i.e., two bogolons) is thus generated from one trigger polariton, as long as the negative delay is within the polariton dephasing time. One of them contributes to the normal branch emission and the other to the ghost branch emission, in the FWM direction $-k$. Energy conservation is maintained because particles are also expected on the ghost branch at the trigger wave vector $k$.

If $\mathrm{NB}$ and $\mathrm{GB}$ are observed on both delay sides, one observes as well some differences in the evolution of the FWM spectrum: At negative delays, the FWM spectrum remains unchanged except for the intensity decay. Since the FWM signal appears after the arrival of the second pulse (in this case the pump pulse), it reflects interactions within a strong superfluid. The longer the negative delay between pump and trigger is, the weaker the excitation of the superfluid by the trigger; but, this always results in the formation of Bogoliubov excitations due to the high polariton density in the pump mode. On the contrary, at positive delays, the polariton superfluid is created first and decays until the trigger pulse arrives and induces the FWM process. On this delay side, we can thus observe a small red-shift of the NB emission and a progressive disappearance of the GB as the positive delay increases, revealing the transition of the polariton gas from superfluid to a dilute gas.

Aside from the normal and ghost branches, a third emission line can be observed in the LP fine structure at $1484.6 \mathrm{meV}$. This line, lying between the energies of the NB and GB, is roughly situated at the energy of the low-density polariton 
dispersion $\left(\frac{\hbar^{2} k_{T}^{2}}{2 m_{p o l}}\right)$. The delay dependence of this middle energy line (ML) is similar to the NB one on the negative delay window but it disappears faster (within 3 ps) at positive delays [Fig. 4(a)]. As discussed in the following, we have good clues that this line is a replica of the NB caused by the dynamical red-shift of the dispersion accompanying the polariton decay for each realization of the quantum fluid.

Emission from the upper polaritons $(1488 \mathrm{meV})$ and strong quantum beats on the whole fine structure are also interesting features of Fig. 4. First, in the whole delay dependence, even close to zero delay where the polariton density is maximum, the observed quantum beats show the same period of $1.2 \mathrm{ps}$ for all the emission lines, in agreement with the Rabi energy of our microcavity. This is sound proof that strong coupling is conserved in our experiment even with the high excitation powers required to observe the Bogoliubov transformation. Small dephasings between the beats of the NB, ML, and GB are observed, which are not explained so far but could come from the specific phase relation between the coherent components of Bogoliubov excitations.

What is more, at negative delays, quantum beats are observed on the whole emission and in particular on the three lines of the LP fine structure. Because the excitation pulses spread over both LPs and UPs, the trigger pulse injects a coherent superposition of lower and upper polaritons that oscillates between photon and exciton states during the interpulse delay. The FWM process can only occur if the pump pulse arrives when the trigger excitation is excitonlike to favor interactions, producing these quantum beats in the FWM emission. Conversely, at positive delays, the quantum beats decay fast and they are very weak on the ghost-branch emission even at small delays. One can better understand these features in the framework of the so-called FWM two-quantum regime. Because we probe the FWM signal proportional to $E_{P}^{2} E_{T}^{*}$, the observed dynamics is a two-photon coherence (equally two polariton dynamics). The occurrence of quantum beats with $3.5-\mathrm{meV}$ frequency is due to the superposition of two FWM contributions: one involving two LPs from the quiescent quantum fluid and the other involving one LP and one UP (the energy difference between these two subsystems being 3.5 $\mathrm{meV})$. The weaker contrast of the beats on the ghost branch compared to the NB and ML shows that interactions between UP and LP do not result in the formation of clear Bogoliubov excitations, but rather in the generation of single-particle excitations, emitting only on the LP normal branch and its replica, the ML. This is certainly due to the faster dephasing of UPs compared to LPs.

The decay-time analysis of the different emission lines confirms this interpretation. They are reported in Table I for both

TABLE I. Decay times of the emission lines of the polariton FWM spectrum, extracted from Fig. 4. Resolution of $0.2 \mathrm{ps}$.

\begin{tabular}{lcc}
\hline \hline Decay time (ps) & Positive delay & Negative delay \\
\hline NB & $3(1.2)$ & 2.8 \\
GB & $3.2(1.1)$ & 2.7 \\
ML & 1 & 2.2 \\
UP & 0.6 & 1.7 \\
\hline \hline
\end{tabular}

delay windows. At negative delay times, the FWM evolution corresponds to the dephasing of polaritons from the trigger. We observe values of $2.7 \mathrm{ps}$ for the LP branch and $1.7 \mathrm{ps}$ for the UP branch. This difference of decay times between the polariton branches can be explained by a slightly negative cavity detuning, favoring the photon content of LPs and also by the influence of the inhomogenous broadening on the upper polariton branch due to interaction with the high-momentum exciton states. $^{20}$

At positive delays, the perturbative approach of the FWM response predicts a decay twice as fast as for negative delays since we are dealing with a two-quantum signal. Actually, this reduction is observed for the UP branch and for the ML. However, we measure twice the expected values for the normal- and ghost-branch decay times. In our system featuring a high LP density, the perturbative approach of the FWM process is not valid anymore. We measure here a dephasing time for the polariton quantum fluid longer than that of two isolated polaritons. This feature reminds of the increase of temporal coherence observed for spontaneous condensates and originates from the Bogoliubov transformation of the LP gas. This point will be discussed in the last section of the paper. At positive delays, the quantum beats disappear faster than the main NB and GB contributions. This decay time, indicated in parentheses in Table I, lies between the measured UP and LP coherence times. This corresponds quite well to an interference effect between a FWM component coming from two LPs and another one coming from one LP and one UP.

These emission features can be qualitatively reproduced by computing the time evolution of the coupled exciton-photon nonequilibrium Gross Pitaevskii equations

$$
\begin{aligned}
& i \hbar \dot{\Psi}_{c}(\vec{r}, t)=\left(\epsilon_{c}(\vec{\nabla})+\delta-i \frac{\gamma_{c}}{2}\right) \Psi_{c}+\frac{\tilde{\Omega}_{R}}{2} \Psi_{x}+F(\vec{r}, t), \\
& i \hbar \dot{\Psi}_{x}(\vec{r}, t)=\left(\epsilon_{x}(\vec{\nabla})+g\left|\Psi_{x}(\vec{r}, t)\right|^{2}-i \frac{\gamma_{x}}{2}\right) \Psi_{x}+\frac{\tilde{\Omega}_{R}}{2} \Psi_{c},
\end{aligned}
$$

where $\psi_{C(X)}$ is the photon (exciton) wave function, $\mathrm{F}(\vec{r}, \mathrm{t})$ the two-pulse excitation, $\epsilon_{C(X)}$ is the photon (exciton) kinetic energy, $\gamma_{C(X)}$ the photon (exciton) decay rate, $\delta$ the cavity detuning, $g$ the polariton-polariton interaction constant, and $\tilde{\Omega}_{R}$ the Rabi coupling energy including the saturation:

$$
\tilde{\Omega}_{R}=\Omega_{R}\left(1-\frac{\left|\Psi_{x}(\vec{r}, t)\right|^{2}}{n_{\text {sat }}}\right) .
$$

The kinetic energy parameters have been chosen to reproduce the experimental dispersion of Fig. 3(c). The interaction constant $g$ was set to $0.01 \mathrm{meV}$ as commonly estimated. ${ }^{30}$ Because our calculation does not allow for considering the inhomogeneous broadening of the UP branch, precise consideration of the photon and exciton linewidths is difficult. We thus fixed equal decay rates of $0.2 \mathrm{ps}^{-1}$ for excitons and photons. The only fitting parameters were the intensity of the pulses and the saturation density. The first one was adjusted to be consistent with the experimental excitation power: the maximal exciton density in our calculation is $3.4 \times 10^{10} \mathrm{~cm}^{-2}$. The second one was tuned to a reasonable value of $5 \times 10^{10} \mathrm{~cm}^{-2}$. $^{31}$ 
Because we are below the weak-coupling regime in the calculation, the main effect of saturation is to conserve a LP-UP splitting close to $3.5 \mathrm{meV}$ and thus a single period for the quantum beats along the whole delay dependence. A smaller value of the saturation density would result in the appearance of the bare excitonic resonance between the upper and lower polaritons.

The main features of the experimental results are well reproduced [Fig. 4(b)] such as the presence of the NB and GB for both positive and negative delays, the long FWM decay time at positive delays for high polariton densities, and the appearance of the ML mainly at negative delays. We also observe weaker quantum beats on the GB at positive delays in agreement with the pure LP origin of the process creating the GB.

Still, some features could not be reproduced, revealing the limitation of the model. First, the calculation shows a long decay time for the UP emission at positive delays. This is linked to the long decay of the quantum beats on the LP emission. We indeed perform the calculation with equal coherence times for photons and excitons. A refined model should take into account the inhomogeneous broadening on the upper polariton branch, which would fasten both the UP decay and the damping of the beats at positive delays. ${ }^{32}$ Second, the calculation shows a strong red-shift of the NB with increasing positive delays and a decrease of the GB/NB intensity ratio. This is due to the decay of the polariton density and the change in the nature of the fluid excitations, from Bogoliubov to single-particle excitations. These features are much weaker in the experiment. This may be attributed to a sublinear dependence of the interaction energy with the polariton density due to screening effects. Finally, the splitting of the NB around 4 ps in the experiment could not be reproduced and the origin of the effect is still under investigation.

\section{B. Interactions between lower and upper polaritons}

To investigate further the interactions between lower and upper polaritons, we performed a second FWM experiment, placing a spectral filter ( $2 \mathrm{meV}$ spectral width) in the optical path of the pump laser in order to excite only the lower polariton branch and thus to prevent the formation of LP-UP superpositions. This experiment was performed at a negative detuning of $\delta \approx-2 \mathrm{meV}$, which is reflected by the observed shorter beating period of $T \approx 1 \mathrm{ps}$. At this detuning, the UP and LP lines are spectrally more separated, and thus it is easier to excite the LP branch only by spectral filtering. The FWM delay dependencies with and without spectral filter are presented in Fig. 5. The use of the spectral filter yields a decrease of the excitation power by a factor of $\sim 3$. Therefore, by choosing the highest laser output power, we are in the excitation regime shortly above the threshold for the Bogoliubov excitation. This explains that the GB emission is weaker than the NB emission ${ }^{14}$ (see Fig. 5). In the experiment without filter, the excitation power $\left(5.7 \times 10^{10}\right.$ photons pulse $\mathrm{cm}^{-1} \mathrm{~cm}^{-2}$ was tuned to obtain the same NB-GB splitting as in the experiment with spectral filter.

Two main changes between these two experiments can be seen: The quantum beats which are clearly visible on the $\mathrm{NB}$ and ML in Fig. 5(a) have almost completely vanished in Fig. 5(b). The normal branch beatings display an average

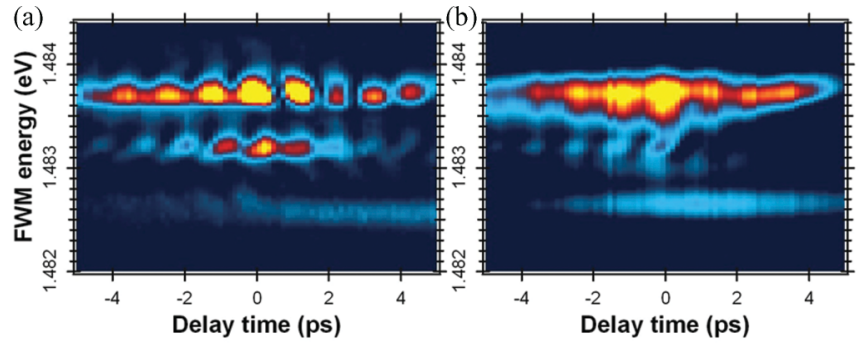

FIG. 5. (Color online) Delay time dependence of the spectrally resolved FWM signal without (a) and with a spectral filter centered on the LP dispersion (b).

contrast of 0.74 in the configuration without spectral filter [Fig. 5(a)], whereas using the filter the average contrast is reduced to 0.27 [Fig. 5(b)]. In contrast, the ghost-branch emission is weakly modified by the excitation change. It suggests that the excitations involving upper polaritons do not show a strong Bogoliubov character and mostly participate to the NB and ML emissions. On top of this, the ML signal in-between the emission of the two Bogoliubov branches almost disappears in the experiment with filter [see Fig. 5(b)]. The ratio between the ML intensity and the NB intensity at $0 \mathrm{ps}$ is reduced by the filter from 0.36 to 0.06 , revealing that the main signal contribution to the ML comes from excitations between lower and upper polaritons. Despite the fact that the ML is a replica of the NB due to time integration, as discussed at length in the last part of the paper, these two lines can still be fed by different processes because they correspond to different regimes of the polariton gas evolution (superfluid for the NB-GB pair and dilute gas for the ML). This special feature is still under investigation. It may be due to the dephasing between lower and upper polaritons, preventing the system for behaving as a single quantum fluid and for generating joint superfluid excitations (stimulated scattering of a pair of LP and UP at $k=0$ onto the superfluid dispersion branches).

\section{Dispersion of a dissipative polariton quantum fluid}

Let us now focus on the dispersion of the FWM response, obtained by varying the excitation angle of the trigger pulse. In Fig. 6, the dispersion is plotted for two positive delays in order to show the effect of the polariton decay and with high excitation intensity $\left(\sim 10^{11}\right.$ photons $/$ pulse $/ \mathrm{cm}^{2}$ for the pump beam). At this excitation intensity, the Bogoliubov transformation is achieved and the superfluid dispersion features the expected shape with normal and ghost branches. As previously observed, ${ }^{14}$ the normal and ghost branches are not symmetric to each other in Fig. 6 and only the NB shows an almost linear behavior. The NB-GB splitting is smaller for the long-delay measurement, showing the effect of the polariton decay on the dispersion.

One notes as well that the middle line, identified previously in the FWM delay dependence, lies along the single-particle dispersion of the microcavity. The coexistence of the Bogoliubov excitation spectrum with the single-particle dispersion in our experiment is surprising. This unlikely comes from the inhomogeneous excitation density as spatial filtering is used to select only the central part of the Gaussian excitation spot. More specifically, this can be explained by the time evolution 


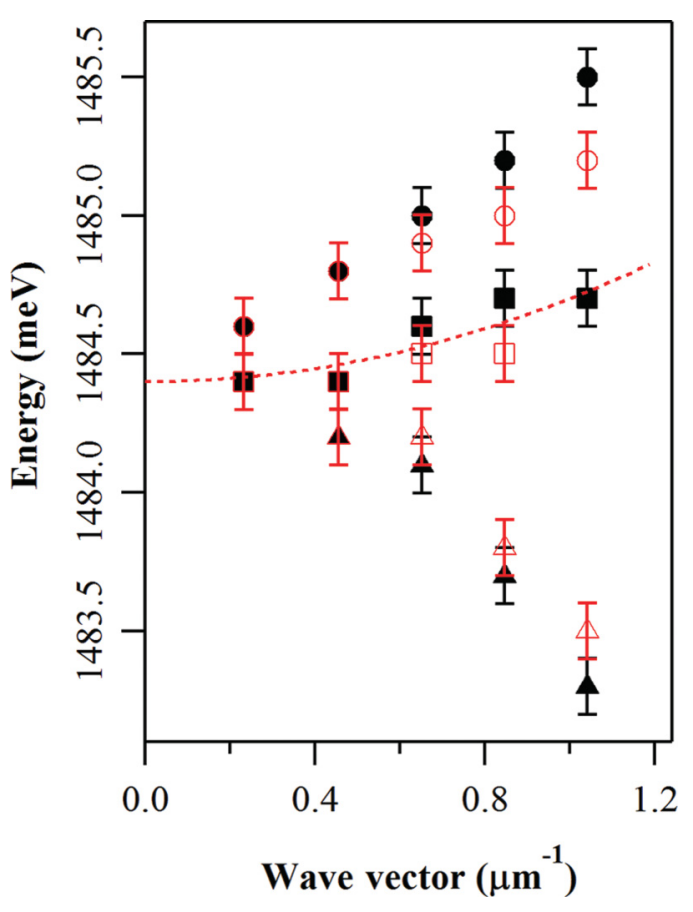

FIG. 6. (Color online) Dispersion of the polariton quantum fluid at short (0.3 ps) positive delay (black markers) and long (3.5 ps) positive delay (red open markers). The NB, ML, and GB are plotted with circles, squares, and triangles, respectively. The experimental fit of the single-particle low-density dispersion is also plotted with dashed line for comparison.

of the system. Let us recall that the measured FWM signal is time integrated. We thus do not resolve the population decay and the transition from superfluid to dilute polariton gas. Lowand high-density spectra should thus appear together in the FWM spectrum. The goal of this section is to enlighten the origin of this unique dispersion, pointing out the strong effect of particle dissipation onto the Bogoliubov spectrum.

Looking at the dispersion relation of a superfluid in the single-particle picture (see Fig. 1), two physical processes can be pointed out: a general blue-shift of the whole dispersion corresponding to the blue-shift of the condensate itself and the coupling of the counterpropagating modes of the Bogoliubov excitations leading to the change of curvature. If the former is linear with the particle density $(g n)$, the latter is sublinear $\left(\sqrt{\left.\left(\frac{\hbar^{2} k^{2}}{2 m}\right)\left(\frac{\hbar^{2} k^{2}}{2 m}+2 g n\right)\right)}\right.$. The change of Bogoliubov-type curvature is therefore less sensitive than the overall energy shift to a decreasing polariton density. Due to our pulsed experiment, we probe a decaying polariton gas which features a dynamical energy shift. In this last part, we demonstrate using semianalytical calculations that the dynamical energy shift is responsible for the asymmetry between the NB and GB and the appearance of the ML in the time-integrated FWM signal.

We solve the time-dependent Schrödinger equation in the lower polariton subspace

$$
i \dot{\Psi}(\vec{r}, t)=\left(\omega(\vec{\nabla})+g|\Psi(\vec{r}, t)|^{2}-i \frac{\gamma}{2}\right) \Psi(\vec{r}, t)+F_{p, t}(\vec{r}, t)
$$

(where $\Psi$ is the LP wave function, $\omega$ its kinetic energy, $g$ the polariton interaction constant, and $F_{p, t}$ the pump and trigger excitations) with the following input wave function:

$$
\Psi(\vec{r}, t)=\varphi_{0}(\vec{r}, t)\left(1+u(\vec{r}, t) e^{i \vec{k} \vec{r}}+v^{*}(\vec{r}, t) e^{-i \vec{k} \vec{r}}\right),
$$

where $\varphi_{0}$ describes the condensate mode at $k=0$ and in analogy with Bogoliubov's theory $u$ and $v *$ are the counterpropagating perturbative modes coupled by polariton interaction. The trigger pulse excites the $v^{*}$ mode and FWM is generated on the $u$ mode.

The master equations of the polariton modes $\varphi_{0}, u$, and $v$ can be written with the operator $\mathcal{L}$ around the condensate solution as $i \dot{\psi}=\mathcal{L} \psi+F_{p, t}^{\prime}$ expressed in the basis $\left(\varphi_{0}, u, v\right)$, with

$$
\mathcal{L}=\left(\begin{array}{ccc}
-i \frac{\gamma}{2}+g\left|\varphi_{0}\right|^{2} & 0 & 0 \\
0 & \omega_{k}+g\left|\varphi_{0}\right|^{2} & g \varphi_{0}^{2} \\
0 & -g \varphi_{0}^{* 2} & -\omega_{k}-g\left|\varphi_{0}\right|^{2}
\end{array}\right)
$$

and $F_{p, t}^{\prime}$ being normalized by $\varphi_{0}$ on the trigger mode. In this form, the dynamical blue-shift is separated from the Bogoliubov transformation since the latter appears in the $(u, v)$ subspace while the former is associated with $\varphi_{0}$. Let us note that the FWM field is given by the product $\varphi_{0} u$. It should also be noted that we assume to have the same damping times for the different modes. From this last equation, one can perform numerical calculation of the FWM spectrum including or removing the dynamical energy shift of the condensate.

In Figs. 7(a), 7(b), and 7(c), the calculated polariton fluid dispersions are displayed for different damping terms. In order to study the effect of dissipation on the excitation spectrum, we increased the damping terms from Fig. 7(a) (negligible damping) to 7(c) (very high damping). Each spectrum 7(a), $7(\mathrm{~b})$, and $7(\mathrm{c})$ is compared with the calculation excluding the dynamical energy shift (Figs. 7(d), 7(e), and 7(f) respectively). The red curves in the different figures display the Bogoliubov dispersion $(\mathrm{NB}+\mathrm{GB})$ for the maximal polariton density and the blue curves display the single-particle parabolic dispersion, which is observed at low polariton densities, and its mirrored parabola on the negative energy side, which is displayed to simplify the following discussion.

The calculations were performed for the following damping terms: $\gamma / g n_{0}=0.01$ [Figs. $7\left(\right.$ a) and $7(\mathrm{~d})$ ], $\gamma / g n_{0}=0.03$ [Figs. 7(b) and 7(e)], and $\gamma / g n_{0}=0.1$ [Figs. 7(c) and 7(f)]. The same excitation conditions were used for the different calculations. In the case of negligible damping (Figs. 7(a) and 7(d)], both spectra show a symmetric behavior for the NB and GB. Their dispersion is clearly Bogoliubov type. Due to the negligible damping, the observed blue-shift at $k=0$ is close to the maximal blue shift in Fig. 7(a).

For higher damping terms, the asymmetry between the two Bogoliubov branches clearly appears and it is accompanied by replicas appearing in the spectra below the NB of Figs. 7(b) and 7 (c). These replicas are the result of the spectral resolution allowing interferences between emission contributions at different times and thus different energies. In particular, for high damping, replicas close to the single-particle dispersion are clearly observed, explaining the appearance of the ML in our experiments. The increasing damping is connected with 

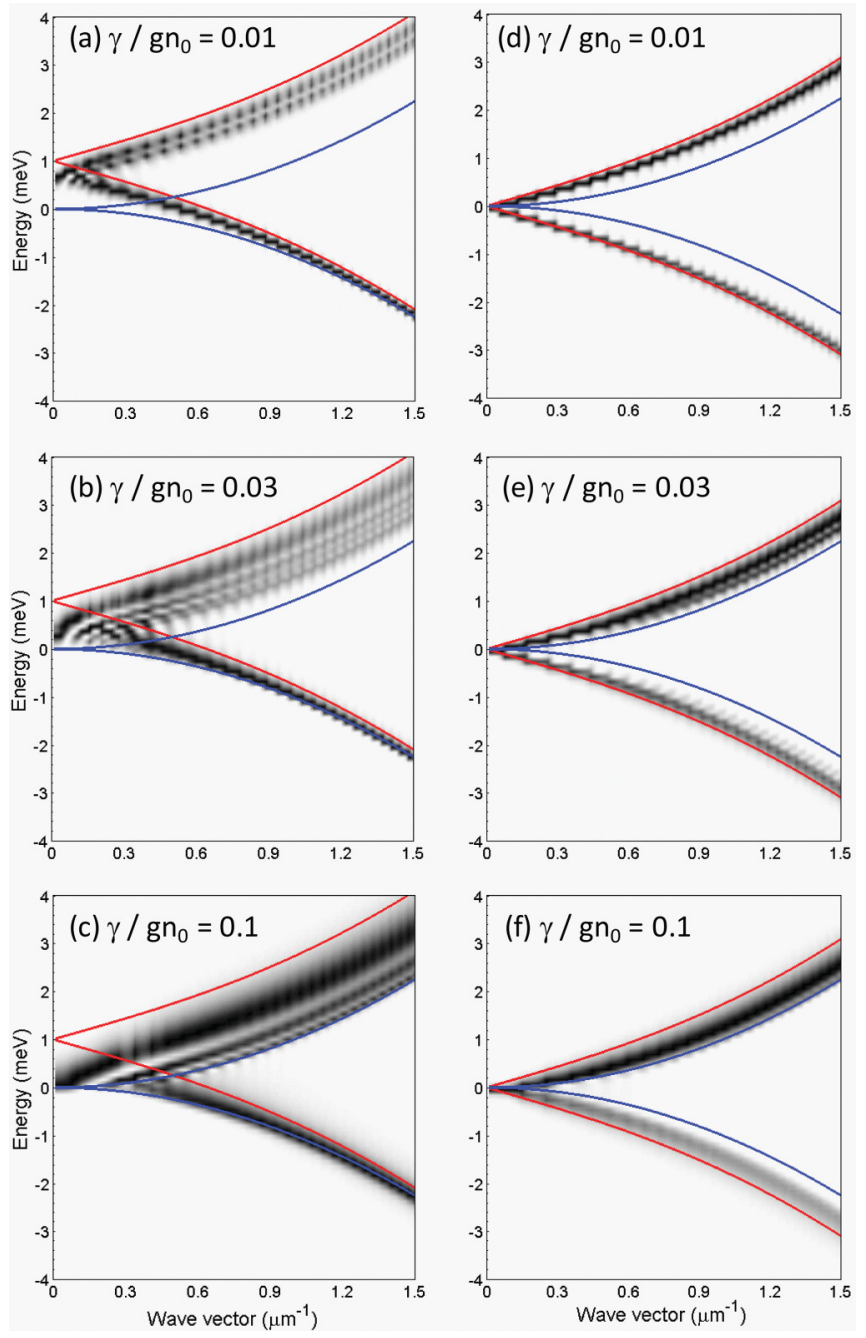

FIG. 7. (Color online) Theoretical dispersion of a coherent polariton gas with and without dynamical blue-shift: Figures (a), (b), and (c) include the dynamical blue-shift and show the change to the asymmetric behavior in the dispersion with increasing damping. The upper red curve displays the NB and GB dispersions at the starting point of the time-integrated measurement $t_{i n t}=0$ and the lower blue curve displays the single-particle parabolic dispersion with its mirrored parabola. In (d), (e), and (f), the dispersion is shown without dynamical blue-shift for different damping rates. Here, the normal and ghost branches are symmetric and the replica have vanished. The slope of the NB in the figures without dynamical blue-shift is less steep than the corresponding one with dynamical blue-shift. The figure is taken from the additional information of Ref. 14.

a decrease of the $k=0$ blue-shift. The NB dispersion slope thus appears to be steeper at low $k$ for higher damping and therefore causes a seeming higher sound velocity as observed experimentally. ${ }^{14}$ However, in Figs. 7(e) and 7(f), where the dynamical blue-shift is excluded, the NB and GB are still symmetric and no replica are present. This confirms that these two effects are caused by the time integration over the damped system.

Additionally, the operator $\mathcal{L}$ provided by Eq. (10) allows us to explain the change in the delay dependence of the FWM field at positive delays due to the Bogoliubov transformation. Using Laplace transform on the $(u, v)$ subspace, one can calculate the temporal response of the FWM field and its dependence on the condensate field

$$
E_{\mathrm{FWM}}=\varphi_{0} u \sim F_{t} \frac{g\left|\varphi_{0}\right|^{2}}{\omega_{B}} \sin \left(\omega_{B} t\right) .
$$

For small $k$, the Bogoliubov frequency $\omega_{B}$ goes as $\sqrt{g\left|\varphi_{0}\right|^{2}} k$ and then the FWM field is linearly dependent on the condensate field. For high $k, \omega_{B} \sim \frac{k^{2}}{2 m}$ and we recover the quadratic dependence to the condensate field, typical of the perturbative FWM regime. This is one more consequence of the interplay between Bogoliubov transformation and particle decay in this new class of quantum fluid that are polaritons.

\section{CONCLUSION}

We have demonstrated that the understanding of a polariton quantum fluid goes beyond the simple Bogoliubov transformation. The unique properties of polaritons, such as their short lifetime and the coexistence of lower and upper polariton modes, modify drastically the excitation spectrum of a polariton quantum fluid. Dissipation results in an asymmetry between the normal and the ghost branches of the Bogoliubov dispersion and also in the appearance of low-energy replicas of the normal branch related to the transition towards the single-particle dispersion with decreasing polariton density. Excitations involving upper polaritons show up during the first ps of the dynamics. Populating mainly the normal branch and its replica (the ML) in the LP dispersion, the UP-LP excitations do not behave as Bogoliubov excitations. Further investigation will be carried out to understand more deeply the nature of the interactions between LPs and UPs in the regime of quantum fluids.

\section{ACKNOWLEDGMENTS}

We acknowledge R. Houdré and U. Oesterle for providing the sample and financial support from SNSF through Quantum Photonics NCCR, Latsis Foundation, and Polatom network. *yoan.leger@insa-rennes.fr

${ }^{1}$ N. N. Bogoliubov, J. Phys. (USSR) 11, 23 (1947).

${ }^{2}$ C. Raman, M. Kohl, R. Onofrio, D. S. Durfee, C. E. Kuklewicz, Z. Hadzibabic, and W. Ketterle, Phys. Rev. Lett. 83, 2502 (1999).

${ }^{3}$ A. Amo, J. Lefrére1, S. Pigeon, C. Adrados, C. Ciuti, I. Carusotto, R. Houdré, E. Giacobino, and A. Bramati, Nat. Phys. 5, 805 (2009).
${ }^{4}$ A. Amo, D. Sanvitto, F. P. Laussy, D. Ballarini, E. del Valle, M. D. Martin, A. Lemaître, J. Bloch, D. N. Krizhanovskii, M. S. Skolnick, C. Tejedor, and L. Viña, Nature 457, 291 (2009).

${ }^{5}$ J. R. Abo-Shaeer, C. Raman, J. M. Vogels, and W. Ketterle, Science 292, 476 (2001). 
${ }^{6}$ K. W. Madison, F. Chevy, W. Wohlleben, and J. Dalibard, Phys. Rev. Lett. 84, 806 (2000).

${ }^{7}$ K. G. Lagoudakis, M. Wouters, M. Richard, A. Baas, I. Carusotto, R. André, Le Si Dang, and B. Deveaud-Plédran, Nat. Phys. 4, 706 (2008).

${ }^{8}$ D. Sanvitto, F. M. Marchetti, M. H. Szymańska, G. Tosi, M. Baudisch, F. P. Laussy, D. N. Krizhanovskii, M. S. Skolnick, L. Marrucci, A. Lemaître, J. Bloch, C. Tejedor, and L. Viña, Nat. Phys. 6, 527 (2010).

${ }^{9}$ J. Steinhauer, R. Ozeri, N. Katz, and N. Davidson, Phys. Rev. Lett. 88, 120407 (2002).

${ }^{10}$ D. S. Jin, J. R. Ensher, M. R. Matthews, C. E. Wieman, and E. A. Cornell, Phys. Rev. Lett. 77, 420 (1996).

${ }^{11}$ R. Ozeri, N. Katz, J. Steinhauer, and N. Davidson, Rev. Mod. Phys. 77, 187 (2005).

${ }^{12}$ J. M. Vogels, K. Xu, C. Raman, J. R. Abo-Shaeer, and W. Ketterle, Phys. Rev. Lett. 88, 060402 (2002).

${ }^{13}$ S. Utsunomiya, L. Tian, G. Roumpos, C. W. Lai, N. Kumada, T. Fujisawa, M. Kuwata-Gonokami, A. Löffler, S. Höfling, A. Forchel, and Y. Yamamoto, Nat. Phys. 4, 700 (2008).

${ }^{14}$ V. Kohnle, Y. Leger, M. Wouters, M. Richard, M. T. Portella-Oberli, and B. Deveaud-Pledran, Phys. Rev. Lett. 106, 255302 (2011).

${ }^{15}$ J. Keeling and N. G. Berloff, Phys. Rev. Lett. 100, 250401 (2008).

${ }^{16}$ K. G. Lagoudakis, F. Manni, B. Pietka, M. Wouters, T. C. H. Liew, V. Savona, A. V. Kavokin, R. Andre, and B. Deveaud-Pledran, Phys. Rev. Lett. 106, 115301 (2011).

${ }^{17}$ G. Grosso, G. Nardin, F. Morier-Genoud, Y. Leger, and B. DeveaudPledran, Phys. Rev. Lett. 107, 245301 (2011).

${ }^{18}$ M. Wouters, arXiv:1105.3597.
${ }^{19}$ J. Kasprzak, M. Richard, S. Kundermann, A. Baas, P. Jeambrun, J. M. J. Keeling, F. M. Marchetti, M. H. Szymańska, R. André, J. L. Staehli, V. Savona, P. B. Littlewood, B. Deveaud, and Le Si Dang, Nature (London) 443, 409 (2006).

${ }^{20}$ V. Savona, J. Phys.: Condens. Matter 19, 295208 (2007).

${ }^{21}$ L. Pitaevskii and S. Strindgari, Bose-Einstein Condensation (Clarendon, Oxford, 2003), p. 56.

${ }^{22}$ M. Wouters and I. Carusotto, Phys. Rev. Lett. 99, 140402 (2007).

${ }^{23}$ C. Ciuti and I. Carusotto, Phys. Status Solidi B 242, 2224 (2005).

${ }^{24}$ M. Wouters and I. Carusotto, Phys. Rev. B 79, 125311 (2009).

${ }^{25}$ G. Nardin, K. G. Lagoudakis, M. Wouters, M. Richard, A. Baas, R. Andre, L. S. Dang, B. Pietka, and B. Deveaud-Pledran, Phys. Rev. Lett. 103, 256402 (2009).

${ }^{26}$ K. L. Hall, G. Lenz, E. P. Ippen, and G. Raybon, Opt. Lett. 17, 874 (1992).

${ }^{27}$ B. Patton, U. Woggon, and W. Langbein, Phys. Rev. Lett. 95, 266401 (2005).

${ }^{28}$ This convention is opposite to the normal time ordering condition of standard FWM experiments in systems such as quantum wells and quantum dots.

${ }^{29}$ R. P. Stanley, R. Houdré, U. Oesterle, M. Gailhanou, and M. Ilegems, Appl. Phys. Lett. 65, 1883 (1994).

${ }^{30}$ C. Ciuti, V. Savona, C. Piermarocchi, A. Quattropani, and P. Schwendimann, Phys. Rev. B 58, 7926 (1998).

${ }^{31}$ D. Sarchi, M. Wouters, and V. Savona, Phys. Rev. B 79, 165315 (2009).

${ }^{32}$ G. Bogiovanni, A. Mura, F. Quochi, S. Gurtler, J. L. Staehli, F. Tassone, R. P. Stanley, U. Oesterle, and R. Houdre, Phys. Rev. B 55, 7084 (1997). 